\title{
New in 2009
}

\author{
As we enter our fifth year of publication, Nature Chemical Biology offers new content and functionality for the \\ chemical biology community.
}

N ature Chemical Biology was launched in 2005 with the primary aims of publishing the highest quality original research and commentary at the interface of chemistry and biology and serving as a central resource for the chemical biology community. Over the years, we have continually sought feedback to ensure that the journal's scope and goals were in line with those of the chemical biology community and to find better ways to deliver our content to our readership. In this spirit, we highlight the new features and functionality that we will be offering in 2009 as we start the fifth volume of Nature Chemical Biology.

Editorial scope. Chemical biology originated with chemists who saw the potential of applying small molecules and chemical tools, particularly those of organic chemistry, toward biological problems. Since then, chemical biology has attracted the attention of inorganic, physical and analytical chemists as well as biologists with research interests spanning all of biology. Chemical biology has not only embraced this larger cross-section of researchers, but in recent years it has educated the first generation of scientists who refer to themselves as 'chemical biologists'. To reflect this broader view of chemical biology, at the launch of the journal the scope of Nature Chemical Biology was defined to encompass all areas of research at the interface of chemistry and biology (Nat. Chem. Biol. 1, 3, 2005). Since then, our interactions with chemical biologists and the evolving research landscape have allowed us to refine the editorial scope of the journal; our new scope statement mirrors the community view that chemical biology today is more broadly integrated across chemistry, biology and allied disciplines, and in the most general terms, seeks to understand and manipulate biological systems with molecular precision (http://www. nature.com/nchembio/authors/index.html\#aim). We welcome submissions in any area at the chemistry-biology interface, particularly those that hybridize chemistry and biology in new ways and present conceptual or practical advances that are likely to open new research frontiers. Further, as the journal continues to evolve with the field, we are always open to discourse with our potential authors and readership regarding the scope of the journal, the content of potential submissions and the rationale behind our editorial decisions (Nat. Chem. Biol. 4, 715, 2008).

More research highlights. To date, each issue of Nature Chemical Biology has included four 'research highlights' featuring papers published in other journals that have caught the interest of the journal's editors. These pieces provide a means to draw attention to studies of broad interest to chemical biologists through short summaries that, we hope, inspire readers to become more aware of diverse topics across chemical biology. On the basis of positive feedback on this section from our readers, we are expanding our monthly Research Highlights section to two printed pages (see pp. 12-13), which will provide more space to highlight a wider range of chemical biology papers each month.

Primers. In a highly interdisciplinary field such as chemical biology, scientists are often stimulated by the diversity of chemical and biological topics that they encounter in chemical biology journals. However, with the limited time that any scientist has, it is often challenging to become conversant in new topics. Commentaries, reviews and perspectives help to quickly broaden our understanding by providing overviews while also being readable and accessible to the nonspecialist. However, we envisioned that it would also be useful to have a quick reference guide to topics in chemical biology, particularly those that are frequently featured in the journal. Starting this year, we will be publishing a series of peer-reviewed 'primers', which are one-page summaries of chemical or biological topics designed to jump-start your ability to comprehend research in a particular area. Available as a tear-out sheet in print issues and also as a PDF download, these primers can be posted on your bulletin board or used as bookmarks for easy reference. We welcome your suggestions for topics to feature in our Primer section.

Enhanced online functionality. Because many chemical biology studies contain chemical compounds, Nature Chemical Biology has already integrated several online resources to enable our readers to better access and use chemical information in published papers (Nat. Chem. Biol. 3, 297, 2007). Beginning with this issue, readers will see further enhancements to the online chemistry functionality of Nature Chemical Biology papers. For example, readers will now be able to visualize the structure of a compound simply by hovering over a bolded compound number in the online version of the manuscript (see for example, http://www.nature.com/nchembio/ journal/v5/n1/full/nchembio.128.html). To improve browsing capability, we have created a 'compound roundup page' that provides access to a full list of the chemical compounds that appear in that month's issue. In parallel with the launch of Nature Chemistry, readers can look for more improvements in our chemical compound functionality, including linking to additional chemistry resources, a richer database of information on each compound, enhanced rendering of three-dimensional compound structures, and more file formats for chemical information.

The 2009 Nature Chemical Biology symposium. Following on two successful symposia on frontier topics at the interface of chemistry and biology, we are pleased to announce the $3^{\text {rd }}$ Nature Chemical Biology symposium, entitled "Chemical Biology in Drug Discovery." The conference, which will be held September 19-20, 2009 in Cambridge, Massachusetts, will bring together leaders who use innovative combinations of chemistry and biology to facilitate drug discovery and to expand druggable space. Visit our website (http://www.nature.com/natureconferences/nchembio2009/index.html) to find out more information and sign up for email updates.

We hope that you will find our new content and functionality useful in your research and educational efforts in 2009. We continue to look to you for feedback on how we can make the journal more interesting and informative for the chemical biology community. 